\title{
Impacto da Pandemia de Covid-19 Sobre a Prática de Exercícios Físicos em Mulheres
}

\section{Impact of the Covid-19 Pandemic on the Practice of Physical Exercise in Women}

\author{
Joel Saraiva Ferreira ${ }^{1}$ \\ Tayla Campagna de Assis ${ }^{2}$ \\ Rafael Presotto Vicente $\mathrm{Cruz}^{3}$ \\ Rodolfo André Dellagrana ${ }^{1}$
}

\section{RESUMO}

Objetivo: Avaliar o impacto da pandemia de COVID-19 sobre a prática de exercícios físicos em mulheres, durante o período de distanciamento social estabelecido pelo poder público municipal de Campo Grande/MS. Metodologia: Realizouse um estudo transversal, com abordagem quantitativa e amostragem probabilística por conglomerado, considerando a proporção de mulheres com idade igual ou superior a 18 anos, residentes nas sete regiões urbanas do município. A coleta de dados ocorreu com uso de formulário online, distribuído por aplicativo de mensagem às mulheres cadastradas no programa de promoção de atividades físicas da Fundação Municipal de Esportes, no período de 7 a 14 de abril de 2020. Foram coletados dados sociodemográficos (idade e escolaridade) e quantidade semanal (minutos) de prática de exercícios físicos antes e durante o período de distanciamento social. Os dados foram analisados no programa SPSS versão 20.0, por meio de regressão logística e teste do qui-quadrado. O estudo foi realizado com aprovação do Comitê de Ética em Pesquisa com Seres Humanos da Universidade Federal de Mato Grosso do Sul. Resultados: Foram recebidos 1188 formulários com respostas válidas. Percebeu-se que o número de mulheres ativas fisicamente ( $\geq 150$ minutos/semanais) passou de $51 \%$ para $10,6 \%$, comparando o momento anterior e durante a pandemia de COVID-19 $(p<0,05)$. O período de distanciamento social aumentou oito vezes a chance da população avaliada se tornar insuficientemente ativa fisicamente $(p<0,05)$. Conclusão: Medidas de distanciamento social, destinadas à contenção da COVID-19, provocaram diminuição drástica na proporção de mulheres ativas fisicamente nesse período.

\section{DESCRITORES}

Exercício físico. Infecções por Coronavirus. Isolamento Social.

\begin{abstract}
Objective: to evaluate the impact of the COVID-19 pandemic on the practice of physical exercise in women, during the period of social distancing established by the municipal government of Campo Grande/MS. Methodology: A cross-sectional study with quantitative analysis and probabilistic sampling by conglomerate was performed, considering the proportion of women aged 18 years or over, living in the seven urban regions of the municipality. Data collection took place using an online form, distributed via a message application to women registered in the Municipal Sports Foundation's physical activity promotion program, from April 7 to 14,2020 . The variables analyzed included sociodemographic data (age and schooling) and physical exercise performed weekly (minutes) during social distancing period. Data were analyzed in the software SPSS version 20.0, in which was used a chi-square test and logistic regression. The present study was approved by Human Research Ethics Committee of the Federal University of Mato Grosso do Sul. Results: 1188 forms were received with valid responses. The number of physically active women $(\geq 150$ minutes/week) went from $51 \%$ to $10.6 \%$, comparing the previous moment and during the COVID-19 pandemic $(p<0.05)$. The period of social distancing increased in eight times the chance of the assessed population to become insufficiently physically active $(p<0.05)$. Conclusion: the measures of social distance, aimed at containing COVID-19, caused a drastic decrease in the proportion of women physically active in this period.
\end{abstract}

\section{DESCRIPTORS}

Exercise. Coronavirus Infections. Social Isolation.

${ }^{1}$ Docente da Faculdade de Educação da Universidade Federal de Mato Grosso do Sul, Campo Grande, Mato Grosso do Sul, Brasil.

${ }^{2}$ Secretaria Municipal de Educação de Campo Grande e Secretaria Estadual de Educação de Mato Grosso do Sul, Campo Grande, Mato Grosso do Sul, Brasil.

${ }^{3}$ Centro Universitário UNIGRAN e Fundação Municipal de Esportes de Campo Grande, Campo Grande, Mato Grosso do Sul, Brasil. 
No os dois primeiros meses do ano de 2020 ocorreu uma rápida disseminação da COVID-19 pelo mundo, desde quando os primeiros casos foram identificados na Cidade de Wuhan, na China, no final de 2019. Essa doença, causada por uma nova variação de Coronavírus (SARS-CoV-2), caracterizase pelo quadro febril, tosse seca, dor de garganta, coriza e dificuldade respiratória, dentre outros sinais e sintomas ${ }^{1}$.

Logo após a notificação dos primeiros casos de COVID-19, ainda no final de 2019, a Organização Mundial da Saúde (OMS) já alertava para uma possível condição grave, com consequências ainda pouco conhecidas. Não tardou para que a situação fosse classificada pela OMS como uma emergência de saúde internacional e, em seguida, a condição de pandemia já estava estabelecida².

No Brasil a COVID-19 foi notificada pela primeira vez na Cidade de São Paulo, no dia 25 de fevereiro de $2020^{3}$. Não muito distante dessa data, a doença foi notificada pela primeira vez no Estado de Mato Grosso do Sul, Região Centro-Oeste do Brasil, especificamente, com dois casos na Cidade de Campo Grande, Capital do Estado, no dia 14 de março de $2020^{4}$.

As medidas adotadas para conter o rápido avanço no número de casos de COVID-19 incluíram medidas de isolamento, quarentena ou distanciamento social ${ }^{5}$. Com isso, grande parte da população passou a permanecer consecutivos dias em seus domicílios, mudando inúmeros hábitos pessoais. A prática de exercícios físicos foi um dos hábitos que sofreu alterações durante a pandemia de COVID-19, sendo que em Campo Grande/MS o poder público municipal decretou o fechamento de diversos estabelecimentos, incluindo academias (públicas e privadas) que promoviam a prática regular de exercícios físicos, assim como a suspensão das atividades sistemáticas ou eventuais de esporte, recreação e lazer, realizadas nos diversos equipamentos esportivos do município ${ }^{6}$.

Nesse novo cenário, criado para proteger a população e evitar um possível colapso no sistema de saúde, manter a prática regular de atividades físicas se tornou um desafio, mesmo que incentivado por instituições ligadas à saúde ${ }^{7}$. Assim, a abrangência das alterações impostas pelas medidas de distanciamento social ainda é desconhecida em relação às variáveis ligadas à quantidade de prática de exercícios físicos em alguns grupos populacionais. $\mathrm{Na}$ localidade estudada existia uma frequência menor de mulheres ativas fisicamente, antes da pandemia de COVID-198, quando comparadas com o sexo masculino, o que as torna mais vulneráveis aos elementos que podem causar diminuição na prática habitual de atividades físicas.

Diante deste contexto e considerando que a prática de exercícios físicos pode ser um importante aliado na manutenção da saúde durante a pandemia, devido o seu efeito protetor nos sistemas cardiovascular, neuromuscular, metabólico e endócrino ${ }^{9}$, o presente estudo foi realizado com o objetivo de avaliar o impacto da pandemia de COVID-19 sobre a prática de exercícios físicos em mulheres, durante o período de distanciamento social estabelecido pelo poder público municipal de Campo Grande/MS. 


\section{METODOLOGIA}

Trata-se de um estudo transversal, com abordagem quantitativa dos dados. Devido às limitações logísticas em função da pandemia de COVID-19, com restrição à locomoção urbana e medidas de distanciamento social vigentes no período de realização do estudo, a coleta de dados ocorreu por meio de formulário online vinculado ao Google Forms $₫$, distribuído via aplicativo de mensagem. Dados sociodemográficos e informações a respeito da prática de exercícios físicos (antes e durante a pandemia) foram coletados no período de 7 a 14 de abril de 2020 .

Os dados sociodemográficos foram categorizados previamente: faixa etária (adultas jovens = 18 a 39 anos; adultas de meia idade $=40$ a 59 anos; idosas $=60$ anos ou mais) e escolaridade (ensino fundamental incompleto; ensino fundamental completo; ensino médio completo; ensino superior completo). As informações referentes aos exercícios físicos incluíam a quantidade de prática semanal (em minutos) antes da pandemia e as barreiras percebidas para manter uma rotina de exercícios físicos durante o período de distanciamento social.

Para classificar as participantes do estudo quanto ao nível de atividade física, os dados foram dicotomizados, adotando-se como ponto de corte o valor de 150 minutos/ semanais de atividades físicas de intensidade moderada ou vigorosa, praticadas por pelo menos 10 minutos contínuos em cada sessão. Valores abaixo do ponto de corte receberam a classificação "insuficientemente ativas", enquanto as demais foram identificadas como "ativas fisicamente". Este procedimento se alinha às recomendações da Organização Mundial da Saúde ${ }^{10}$ e a nomenclatura proposta por Dumith ${ }^{11}$.

A população foi constituída por mulheres com idade igual ou superior a 18 anos, residentes na área urbana do Município de Campo Grande/MS, cadastradas na Fundação Municipal de Esportes (FUNESP) como participantes do programa "Movimenta Campo Grande", que oferece gratuitamente prática de exercícios físicos à população campo-grandense, em todas as regiões urbanas da cidade.

Utilizou-se amostragem probabilística por conglomerado (sete regiões urbanas do município). O plano amostral foi definido considerando uma população finita de 276 mil pessoas, com margem de erro de $3 \%$, nível de confiança de $95 \%$ e distribuição estimada de resposta de $50 \%$. O resultado indicou a necessidade de incluir ao menos 1.063 mulheres na amostra.

Os critérios de inclusão estabelecidos para as participantes do estudo foram: a) praticar exercícios físicos regularmente antes das medidas de distanciamento social, em função da pandemia de COVID-19; b) ter no mínimo 18 anos de idade no momento da coleta de dados; c) responder todas as questões do formulário online no prazo estabelecido para o estudo.

A tabulação dos dados ocorreu em planilha do programa Microsoft Excel®, gerada a partir do formulário online original (Google Forms ${ }^{\circledR}$ ). Para a análise dos dados utilizou-se incialmente a estatística descritiva (frequências absolutas e relativas) para identificar a proporção de barreiras para a manutenção da prática de exercícios físicos 
e nível de atividade física antes e durante a pandemia de COVID-19. O teste Quiquadrado foi utilizado para a comparação entre faixas etárias e escolaridade, nas proporções das barreiras para a prática de exercício físico e no nível de atividade física antes e durante a pandemia. Análise de regressão logística binária foi realizada para estimar a relação do baixo nível de atividade física com o distanciamento social antes e durante a pandemia. Os cálculos estatísticos foram desenvolvidos no programa estatístico SPSS, versão 20.0, adotando um nível de significância de $5 \%$.

O presente estudo integra a pesquisa "Observatório de atividades físicas e esportivas", aprovado pelo Comitê de Ética em Pesquisa com Seres Humanos, vinculado à Universidade Federal de Mato Grosso do Sul, sob o protocolo $n^{\circ}$. 3.416.611.

\section{RESULTADOS}

Foram coletados 1.188 formulários com respostas válidas, suficientes para atender o plano amostral da pesquisa. Ocorreu proporcionalidade entre a quantidade de respondentes e de habitantes em cada conglomerado do estudo (sete regiões urbanas do município). A maior prevalência foi de mulheres de meia-idade (54\%) e com ensino médio completo $(47,1 \%)$.

A quantidade de exercícios físicos praticados semanalmente pelas participantes do estudo foi diferente nos momentos que precederam as medidas de distanciamento social e durante o período de vigência dos decretos municipais vinculados a essa finalidade. As mulheres que praticavam ao menos 150 minutos semanais de atividades físicas e, por isso, foram consideradas ativas fisicamente, passaram de $51 \%$ para $10,6 \%$. As que praticavam menos de 150 minutos semanais de atividades físicas, classificadas como insuficientemente ativas fisicamente, passaram de $49 \%$ para $89,4 \%\left(X^{2}=454,900\right.$; $p<0,05$ ) (Figura 1a). No que se refere à manutenção da prática de exercícios físicos durante a pandemia de COVID-19, apenas $8,8 \%$ das mulheres conseguiram manter mais de 150 minutos semanais, enquanto $89,4 \%$ reduziram ou mantiveram os níveis de atividade física abaixo de 150 minutos semanais durante a pandemia (Figura 1b).

$\mathrm{Na}$ análise de associação da faixa etária e escolaridade com o nível de atividade física antes e durante a pandemia (Tabela 1), não foram encontradas associações significativas $(p>0,05)$.

A Tabela 2 apresenta a inter-relação do baixo nível de atividade física com as situações antes e durante a pandemia de COVID-19. Verificou-se que durante a pandemia mulheres têm aproximadamente oito vezes mais chances de apresentarem nível insuficiente de atividade física, em comparação ao período que antecedeu a pandemia $(p<0,05)$.

As participantes do estudo foram questionadas sobre as dificuldades percebidas para manterem-se ativas fisicamente durante o período de distanciamento social. Alguns fatores foram apontados como barreiras para a continuidade da prática de exercícios físicos, sendo o relato mais prevalente a falta de espaço ou local adequado (48,3\%), seguido da falta de orientação de um/uma profissional de educação física $(41,3 \%)$ e, 
Figura 1. Nível de atividade física (NAF) de mulheres e manutenção da prática de atividade física durante a pandemia de COVID-19

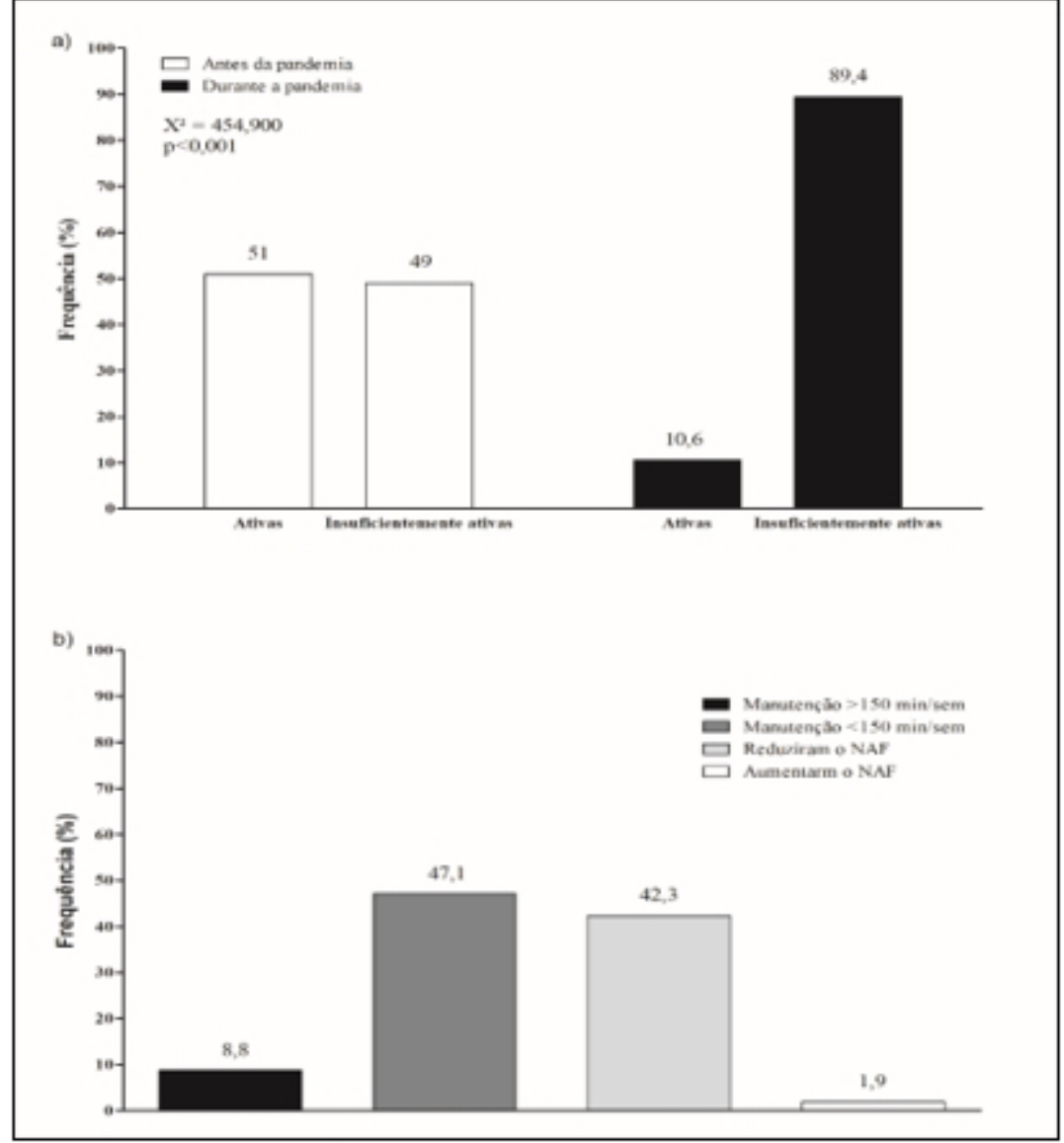

(a) Nivel de atividade física (NAF) de mulheres, antes e durante as medidas distanciamento social, e (b) manutenção da prática de atividade física durante a pandemia de COVID-19.

Tabela 1. Nível de atividade física de mulheres, de acordo com a faixa etária e escolaridade, antes e durante a pandemia de COVID-19

\begin{tabular}{|c|c|c|c|c|c|c|}
\hline \multirow{3}{*}{$\begin{array}{c}\text { Variáveis } \\
\text { Sociodemográficas }\end{array}$} & \multirow{3}{*}{$\mathrm{n}$} & \multicolumn{2}{|c|}{ Antes da pandemia } & \multicolumn{3}{|c|}{ Durante a pandemia } \\
\hline & & $\begin{array}{l}\text { Insuficientem } \\
\text { ente ativos }\end{array}$ & $\begin{array}{c}\text { Ativos } \\
\text { fisicamente }\end{array}$ & $\begin{array}{c}\text { Insuficientement } \\
\text { e ativos }\end{array}$ & $\begin{array}{c}\text { Ativos } \\
\text { fisicamente }\end{array}$ & \multirow[t]{2}{*}{ p-valo } \\
\hline & & $\mathrm{n}(\%)$ & $\mathrm{n}(\%)$ & $\mathrm{n}(\%)$ & $\mathrm{n}(\%)$ & \\
\hline Total & 1188 & $582(49,0)$ & $606(51,0)$ & $1062(89,4)$ & $126(10,6)$ & \\
\hline \multicolumn{7}{|l|}{ Faixa etária } \\
\hline 18 a 39 anos & 394 & $207(35,6)$ & $187(30,9)$ & $353(33,2)$ & $41(32,5)$ & \multirow{3}{*}{0,578} \\
\hline 40 a 59 anos & 654 & $306(52,6)$ & $348(57,4)$ & $580(54,6)$ & $74(58,7)$ & \\
\hline$\geq 60$ anos & 140 & $69(11,9)$ & $71(11,7)$ & $129(12,1)$ & $11(8,7)$ & \\
\hline \multicolumn{6}{|l|}{ Escolaridade } & \multirow{5}{*}{0,774} \\
\hline EF incompleto & 55 & $29(5,0)$ & $26(4,3)$ & $53(5,0)$ & $2(1,6)$ & \\
\hline EF completo & 95 & $50(8,6)$ & $45(7,4)$ & $83(7,8)$ & $12(9,5)$ & \\
\hline EM completo & 569 & $279(47,9)$ & $290(47,9)$ & $502(47,3)$ & $67(53,2)$ & \\
\hline ES completo & 469 & $224(38,5)$ & $245(40,4)$ & $424(39,9)$ & $45(35,7)$ & \\
\hline
\end{tabular}


Tabela 2. Inter-relação do baixo nível de atividade física de mulheres, antes e durante a pandemia de COVID-19

\begin{tabular}{|c|c|c|c|}
\hline & Antes da pa & Durante a pandemia & \\
\hline & & 188 & \\
\hline & Ref. & OR (IC95\%) & p-valor \\
\hline $\begin{array}{l}\text { Nivel de atividade física } \\
\text { Insuficientemente ativos }\end{array}$ & 1 & $8,77(7,07-10,90)$ & $<0,01$ \\
\hline
\end{tabular}

por fim, falta de equipamentos ou aparelhos $(10,4 \%)$. Ademais, foi observada associação significativa entre a faixa etária e as barreiras para a prática de atividade física $\left(X^{2}=10,090\right.$; $p=0,039$ ), sendo que a falta de espaço foi a principal barreira para mulheres na faixa etária de 18 à 59 anos (adultas jovens e adultas de meia idade), enquanto a falta de orientação profissional foi considerada a principal barreira para mulheres idosas (Tabela 3).

\section{DISCUSSÃO}

Esta pesquisa avaliou o impacto da pandemia de COVID-19 sobre a prática de exercícios físicos em mulheres adultas e idosas, durante o período de distanciamento social estabelecido pelo poder público municipal de Campo Grande/MS. Os resultados apontaram para uma drástica diminuição na quantidade de exercícios físicos que essa população passou a realizar semanalmente, comparando o período anterior à pandemia e durante os momentos de distanciamento social.

Para compreender o impacto que a pandemia de COVID-19 ocasionou sobre a prática de exercícios físicos entre o público pesquisado no presente estudo, recorreuse a comparação da quantidade semanal de exercícios físicos realizados em dois momentos: antes e durante a vigência das medidas de distanciamento social. A proporção de mulheres ativas fisicamente passou de $51 \%$ para $10,6 \%$, enquanto

Tabela 3. Barreiras para mulheres manterem a prática de exercícios físicos durante a pandemia de COVID-19

\begin{tabular}{|c|c|c|c|c|c|}
\hline $\begin{array}{c}\text { Variáveis } \\
\text { sociodemográficas }\end{array}$ & $\mathrm{n}$ & $\begin{array}{l}\text { Falta de espaço ou } \\
\text { local adequado }\end{array}$ & $\begin{array}{c}\text { Falta de orientação } \\
\text { profissional }\end{array}$ & $\begin{array}{c}\text { Falta de } \\
\text { equipamentos ou } \\
\text { aparelhos }\end{array}$ & p-valor \\
\hline & & $n(\%)$ & $\mathrm{n}(\%)$ & $\mathrm{n}(\%)$ & \\
\hline Total & 1188 & $574(48,3)$ & $491(41,3)$ & $123(10,4)$ & \\
\hline $\begin{array}{l}\text { Faixa etária } \\
18 \text { a } 39 \text { anos } \\
40 \text { a } 59 \text { anos } \\
\text { ₹ } 60 \text { anos }\end{array}$ & $\begin{array}{l}394 \\
654 \\
140\end{array}$ & $\begin{array}{c}211(53,6) \\
307(46,9) \\
56(40,0)\end{array}$ & $\begin{array}{c}141(35,8) \\
281(43,0) \\
69(49,3)\end{array}$ & $\begin{array}{l}42(10,7) \\
66(10,1) \\
15(10,7)\end{array}$ & 0,039 \\
\hline $\begin{array}{l}\text { Escolaridade } \\
\text { EF incompleto } \\
\text { EF completo } \\
\text { EM completo } \\
\text { ES completo }\end{array}$ & $\begin{array}{c}55 \\
95 \\
569 \\
469\end{array}$ & $\begin{array}{l}25(45,5) \\
42(44,2) \\
262(46,1) \\
245(52,2)\end{array}$ & $\begin{array}{l}27(49,0) \\
46(48,4) \\
242(42,5) \\
176(37,5)\end{array}$ & $\begin{array}{c}3(5,5) \\
7(7,4) \\
65(11,4) \\
48(10,3)\end{array}$ & 0,171 \\
\hline
\end{tabular}

Nota: $\mathrm{EF}=$ Ensino Fundamental; $\mathrm{EM}=$ Ensino Médio; $\mathrm{ES}=$ Ensino Superior . 
as insuficientemente ativas passaram de $49 \%$ para $89,4 \%$, quando comparados os dois momentos $(p<0,05)$. Esses resultados também estão sendo observados em outras regiões do Brasil e parecem ter relação com as restrições à prática de exercícios físicos ao ar livre ${ }^{12,13}$, o que mostra pouca autonomia do grupo pesquisado para se exercitar no contexto domiciliar.

A redução de atividade física, em conjunto com outros fatores de risco, pode agravar as condições de saúde ${ }^{14} \mathrm{e}$, por isso, é preocupante constatar que existe uma relação entre as medidas de distanciamento social e a redução na prática de exercícios físicos ${ }^{15}$. Sendo assim, a recomendação de manutenção do estilo de vida ativo fisicamente, mesmo em ambiente doméstico ${ }^{16}$, é essencial enquanto a pandemia de COVID-19 impedir o acesso das pessoas aos locais específicos de prática de exercícios físicos.

O período em que as mulheres investigadas no presente estudo estiveram cumprindo medidas de distanciamento social, em função da pandemia de COVID-19, aumentou oito vezes as chances de se tornarem insuficientemente ativas fisicamente, em comparação ao período que antecedeu a pandemia $(p<0,05)$. Nessas condições, com redução drástica na prática de exercícios físicos, efeitos negativos podem ser gerados aos sistemas cardiovascular, metabólico, muscular e endócrino ${ }^{9}$, além de estresse crônico com sobrecarga à saúde mental ${ }^{17}$.

As participantes do estudo apontaram as principais barreiras para manter a prática de exercícios físicos durante a pandemia de COVID-19. Todos os motivos narrados se relacionaram com a condição estabelecida durante as medidas de distanciamento social, sendo que a falta de local ou espaço adequado foi mais prevalente entre as mulheres adultas jovens e de meia-idade, quando comparadas com as idosas $(p<0,05)$. Esse tipo de barreira, considerada componente do domínio físico ${ }^{18}$, também foi identificada entre mulheres que recebem atendimento na área da saúde pública $^{19}$, o que reforça a condição de pouca independência para praticar exercícios físicos, por boa parte da população feminina, quando não existe acesso a locais apropriados e específicos para essa finalidade.

Resultados de estudos realizados tanto no Brasil ${ }^{20}$ quanto nos Estados Unidos ${ }^{21}$ já demonstraram que a disponibilidade de locais planejados e construídos para a prática de exercícios físicos, como praças e parques esportivos, têm potencial para tornar a população mais ativa fisicamente. Esse fenômeno parece ter se repetido com a população investigada no presente estudo, que vislumbrou a inacessibilidade aos locais de prática de exercícios como elemento capaz de prejudicar o estilo de vida ativo fisicamente de mulheres adultas.

As idosas avaliadas no presente estudo, quando comparadas com mulheres adultas, apontaram de forma mais acentuada a falta de orientação profissional como a barreira mais prevalente no período estudado $(p<0,05)$. Esta barreira é considerada componente do domínio social ${ }^{18}$ e tem relação com a necessidade percebida pela população mais velha, que requer o auxílio proporcionado por professores ou instrutores na execução correta dos movimentos e também na motivação para a prática dos exercícios físicos ${ }^{22}$.

Sendo assim, não restam dúvidas 
quanto à demanda pelo desenvolvimento de estratégias voltadas ao incentivo da prática de exercícios físicos no contexto domiciliar, enquanto existir algum tipo de restrição à mobilidade urbana para evitar a disseminação da COVID-19. Uma estratégia para essa finalidade pode ser o trabalho remoto de prescrição e acompanhamento dos exercícios físicos, tal como já ocorre em outras áreas da saúde, por meio do telessaúde ${ }^{23}$. Nesse caso, é importante atentar-se para cuidados como o baixo custo e alta acessibilidade que as iniciativas de promoção de atividades físicas devem ter, para não preterir uma parcela da sociedade que já é vulnerável por aspectos socioeconômicos.

Por fim, é válido destacar que o presente estudo apresenta limitações quanto ao potencial de extrapolação dos resultados para outras localidades, por conta de eventuais vieses oriundos da coleta online dos dados e, principalmente, porque a pandemia de COVID-19 pode ter desfechos distintos em condições socioculturais e ambientais diferentes do contexto aqui analisado, como por exemplo, a taxa de distanciamento social, que se mostra heterogênea entre os estados brasileiros ${ }^{24}$. Mesmo assim, a pesquisa atingiu o objetivo proposto e foi capaz de trazer dados iniciais para o auspicioso debate em torno das consequências da pandemia de COVID-19 sobre a prática de exercícios físicos.

\section{CONCLUSÃO}

No conjunto de medidas estabelecidas para proteger a população e evitar um possível colapso do sistema de saúde, o distanciamento social imposto pelo governo municipal de
Campo Grande/MS impactou na diminuição da prática de exercícios físicos entre mulheres que já tinham esse hábito incorporado às suas rotinas antes da pandemia, aumentando em oito vezes a chance de se tornarem insuficientemente ativas.

As medidas de distanciamento social mantiveram as pessoas por mais tempo no contexto domiciliar, o que resultou em barreiras para a manutenção de um estilo de vida fisicamente ativo. Fica assim exposta a demanda por intervenções voltadas à promoção de exercícios físicos no contexto domiciliar durante a pandemia de COVID-19, sendo que no presente estudo a maior dificuldade a ser superada pelo público feminino é a falta de espaço ou local adequado.

Sendo assim, o distanciamento social, considerado uma proteção indispensável para a emergência de saúde pública provocada pela pandemia de COVID-19, trouxe efeitos que merecem acompanhamento mais aprofundado em futuros estudos populacionais, que sejam capazes de identificar nuances ainda inexploradas em relação à drástica diminuição na proporção de pessoas ativas fisicamente durante a pandemia e a elevada chance das pessoas se tornarem insuficientemente ativas enquanto cumprem o distanciamento social indicado pelas autoridades sanitárias.

\section{AGRADECIMENTOS}

Os autores agradecem a Fundação Municipal de Esportes de Campo Grande/ MS (FUNESP) pela viabilidade de acesso à amostra e aos seus Agentes Sociais de Esporte e Lazer pelo encaminhamento dos formulários online aos participantes do estudo. 


\section{REFERÊNCIAS}

1. Guo YR, Cao QD, Hong ZS, Tan YY, Chen SD, Jin HJ, et al. The origin, transmission and clinical therapies on coronavirus disease 2019 (COVID-19) outbreak - an update on the status. Mil Med Res. 2020; 7(11):2-10.

2. World Health Organization (WHO). Coronavírus disease 2019 (COVID-19) - Situation Report Number 67. Geneva: WHO; 2020.

3. Brasil. Ministério da Saúde. Secretaria de Vigilância em Saúde. Centro de Operações de Emergência em Saúde Pública. Boletim Epidemiológico 11 - Semana Epidemiológica 16 (12 - 18/04). Doença pelo coronavírus 2019 (COVID-19). Brasília: Ministério da Saúde; 2020.

4. Mato Grosso do Sul. Secretaria Estadual de Saúde. Boletim coronavírus 4/4/2020. Campo Grande: Secretaria Estadual de Saúde; 2020.

5. Wilder-Smith A, Freedman DO. Isolation, quarantine, social distancing and community containment: pivotal role for old-style public health measures in the novel coronavirus (2019-nCoV) outbreak. J Travel Med. 2020; $1-4$.

6. Campo Grande. Portaria $n^{\circ} 10 / F U N E S P$, de 19 de março de 2020. Dispõe sobre o fechamento temporário dos equipamentos de esporte e lazer, para evitar a disseminação do novo coronavírus - COVID-19 entre seus frequentadores. Campo Grande: Diogrande; 2020.

7. Sociedade Brasileira de Medicina do Exercício e do Esporte. Informe da Sociedade Brasileira de Medicina do Exercício e do Esporte (SBMEE) sobre exercício físico e coronavírus (COVID-19). São Paulo: SBMEE; 2020.

8. Brasil. Ministério da Saúde. Vigitel Brasil 2019: vigilância de fatores de risco e proteção para doenças crônicas por inquérito telefônico. Brasília: Ministério da Saúde; 2020.

9. Narici M, De Vito G, Franchi M, Paoli A, Moro T, Marcolin $\mathrm{G}$, et al. Impact of sedentarism due to the COVID-19 home confinement on neuromuscular, cardiovascular and metabolic health: Physiological and pathophysiological implications and recommendations for physical and nutritional countermeasures. Eur J Sport Sci. 2021; 21(4):614-635.

10. World Health Organization (WHO). Global recommendations on physical activity for health. Geneva: WHO; 2010.
11. Dumith SC. Atividade Física e sedentarismo: diferenciação e proposta de nomenclatura. Rev Bras Ativ Fis Saude. 2010; 15(4):253-254.

12. Bezerra ACV, Silva CEM, Soares FRG, Silva JAM. Fatores associados ao comportamento da população durante o isolamento social na pandemia de COVID-19. Ciênc Saúde Colet. 2020; 25(Supl.1):2411-2421

13. Silva CEM, Cruz Neto CC, Bezerra ACV, Santos RT, Silva JAM. Influência das condições de bem-estar domiciliar na prática de isolamento social durante a Pandemia de COVID-19. J Health Biol Sci. 2020; 8(1):1-7.

14. Owen N, Sparling PB, Healy G, Dunstan DW, Matthews CE. Sedentary behavior: emerging evidence for a new health risk. Mayo Clin Proc. 2010; 85(12):1138-1141.

15. Chen P, Mao L, Nassisn GP, Harmer P, Ainsworth BE, Li F. Coronavirus disease (COVID-19): The need to maintain regular physical activity while taking precautions. J Sport Health Sci. 2020; 9:03-104.

16. Pitanga FJG, Beck CC, Pitanga CPS. Atividade física e redução do comportamento sedentário durante a pandemia de coronavírus. Arq Bras Cardiol. No prelo.

17. Burtscher J, Burtscher M, Millet GP. (Indoor) isolation, stress and physical inactivity: vicious circles accelerated by COVID-19? Scand J Med Sci Sports. 2020; 30:15441545.

18. Vieira VR, Silva JVP. Barreiras à Prática de atividades físicas no lazer de brasileiros: uma revisão sistemática. Pensar Prát. 2019; 22(54448):1-22.

19. Gomes GAO, Papini CB, Nakamura PM, Teixeira IP, Kokubun E. Barreiras para a prática de atividade física entre mulheres atendidas na atenção básica de saúde. Rev Bras Ciênc Esporte. 2019;41(3):263-270.

20. Hino AAF, Rech CR, Gonçalves PB, Reis, RS. Acessibilidade a espaços públicos de lazer e atividade física em adultos de Curitiba, Paraná, Brasil. Cad Saúde Pública. 2019; 35(12):1-12.

21. Kaczynski AT, Besenyi GM, Stanis SAW, Koohsari MJ, Oestman KB, Bergstrom R, et al. Are park proximity and park features related to park use and park-based physical activity among adults? Variations by multiple socio-demographic characteristics. Int J Behav Nutr Phys Act. 2014;11(146):1-14. 
22. Monteiro LZ, Lira BA, Souza P, Braga Junior FD. Barreiras percebidas para a prática de atividade física entre servidores do setor administrativo de uma faculdade do Distrito Federal. Cienc Trab. 2018; 20(62):97-102.

23. Harzheim E, Chueiri PS, Umpierre RN, Gonçalves MR, Siqueira ACS, D'ávila OP, et al. Telessaúde como eixo organizacional dos sistemas universais de saúde do século XXI. Rev Bras Med Fam Comunidade. 2019;14(41):1-9.
24. Índice de isolamento social [Internet]. Mapa brasileiro da COVID-19. Inloco Tech Blog; c2020.

\section{CORRESPONDÊNCIA}

Joel Saraiva Ferreira

Rua Tapés, 33 - Vila Jussara

- Campo Grande/MS

CEP 79092-500

E-mail: joel.ferreira@ufms.br 
